\title{
PERFIL SOCIOECONÓMICO DEL ESTUDIANTE DE EDUCACIÓN SUPERIOR DE HONDURAS EN EL SIGLO XXI
}

\author{
Hugo Rolando Noé Bustamante*
}

DOI: $10.5377 /$ eya.v10i1.9048

Recibido: 4/02/2019 Aceptado: 28/08/2019

\section{RESUMEN}

Los cambios del perfil sociodemográfico y socioeconómico del estudiante de educación superior a nivel global han sido significativos en este nuevo milenio. Destacan, entre otros, la masificación y la diversificación, lo cual a su vez ha ampliado los métodos de enseñanza y los estilos de aprendizaje del estudiante. El objetivo principal de este estudio es presentar el perfil socioeconómico del estudiante de educación superior hondureño de la actualidad; para ello se analizan y siguen algunos de los principales cambios sociodemográficos y socioeconómicos observados a nivel internacional. Además, se compara entre estudiantes hondureños de acuerdo con su modalidad de estudio y se considera su opinión en torno a su futuro laboral/académico. Se consideró la literatura de relevantes publicaciones nacionales e internacionales y se aplicó una encuesta como principal instrumento de recolección de información primaria a una muestra de estudiantes de Instituciones de Educación Superior (IES) de Honduras. Los resultados indican que existe una mayor masificación y diversificación del estudiante de educación superior hondureño, pero en menor escala que en América Latina y otras regiones del mundo. Además, se muestran evidentes diferencias demográficas, económicas y de circunstancias de vida entre los estudiantes de modalidad presencial de pregrado y los de distancia y posgrado. Finalmente, y considerando los cambios en el perfil del estudiante del nuevo milenio, se presentan brevemente algunas modificaciones que han surgido en las modalidades de enseñanza y aprendizaje de este nivel educativo.

Palabras clave: perfil estudiantes, educación superior, modalidades de enseñanza, Honduras

JEL: I230

\footnotetext{
* Docente de la Dirección de Educación Superior (DES) de la Universidad Nacional Autónoma de Honduras (UNAH). Este documento es producto de labores de investigación de la DES y contó con la colaboración del Máster Isaac Toro Trejo para la implementación de la encuesta y el Lic. Henry Osorto en la parte del muestreo. Adicionalmente, se agradecen los comentarios realizados por el Msc. Ramón Salgado y a las autoridades académicas del CEDAC, la UMH, UNAH, UNICAH y UPNFM por su contribución en la implementación de la encuesta. La responsabilidad del contenido de este artículo es de su autor.
} 


\title{
SOCIOECONOMIC PROFILE OF HIGHER EDUCATION STUDENT OF HONDURAS IN THE 21ST CENTURY
}

Hugo Rolando Noé Bustamante

DOI: $10.5377 /$ eya.v10i1.9048

\begin{abstract}
There have been significant changes in the sociodemographic and socioeconomic higher education student profile in this new millennium. Among them, massification and diversification have been notorious, and these in turn have extended new teaching and learning methods. The main objective of this document is to present a profile of the higher education Honduran student according to important sociodemographic and socioeconomic changes observed internationally. Additionally, a brief comparison is made among Honduran students of different learning modalities and the opinions of their academic/working future is considered.

To achieve the main objective, relevant national and international publications were considered and a survey was applied to a sample of students of Higher Education Institutions (HEI) in Honduras. The results indicate that there is greater massification and diversification of the Honduran higher education student, but less than in Latin America and other regions of the world. Also, there are evident differences in demographic, economic, and life circumstances among in person undergraduates, distance learning, and master students. Finally, and considering the changes in the profile of the higher education student, a brief description of new teaching and learning methods is provided.
\end{abstract}

Key words: student profile, higher education, teaching modalities, Honduras

JEL: I230 


\section{INTRODUCCIÓN}

Las primeras dos décadas del siglo XXI han visto cambios sustanciales en el perfil del estudiante de educación superior a nivel global. Entre estos cambios destacan: un aumento considerable en la matricula, diversificación del perfil socioeconómico y demográfico del estudiante, feminización de la matrícula y de graduados, y un incremento en la oferta y demanda de la educación a distancia y virtual.

Cambios similares se manifiestan en Honduras; por ejemplo, la matrícula universitaria aumento 40\% entre 2007 y 2016 en el nivel de licenciatura y 54\% entre el 2006 y el 2015 a nivel de maestría; las mujeres representan una mayoría estudiantil tanto en la matricula (57\% en 2015), como en el total de graduados. Al mismo tiempo, la matrícula de educación a distancia y una mayor cantidad de sedes regionales de las Instituciones de Educación Superior (IES) han permitido un mayor acceso a una mayor diversidad de estudiantes (Dirección de Educación Superior (DES, 2018).

Sin embargo, otros temas enfatizados en la literatura internacional ayudan a complementar el perfil del estudiante de educación superior desde el punto de vista socioeconómico y sociodemográfico. Por ejemplo, presentan información sobre los estudiantes con edades "mayores" a las tradicionales, los que son padres de familia, los que trabajan, etc. Es decir, al denominado estudiante "no tradicional" que crece en número en diferentes regiones del mundo.

El objetivo principal de esta investigación es caracterizar socioeconómicamente al estudiante de educación superior hondureño del nuevo milenio. De esta forma se complementa la información hecha pública recientemente por la Dirección de Educación Superior (DES) en su informe "La educación superior en Honduras y sus líneas estratégicas de desarrollo" (DES, 2018). Adicionalmente, este perfil estudiantil, más característico de este nuevo milenio y una nueva generación de estudiantes, sugiere nuevos estilos de enseñanza (distancia y virtual, por ejemplo) y aprendizaje (medios digitales, redes sociales). En este sentido, uno de los aspectos a investigar en el futuro es el surgimiento y aumento de los cursos en línea, así como el otorgamiento de grados académicos bajo esta modalidad.

Entre los hallazgos de este estudio, se puede decir que, a pesar de una diversificación del estudiante de educación superior en Honduras en el nuevo milenio, no existe un crecimiento del estudiante "no tradicional" de pregrado tan evidente o significativo como en otras regiones del mundo. Los estudiantes que más se acercan a este concepto en el caso hondureño son los de la modalidad a 
distancia y los de posgrado. Además, si bien es cierto hay una leve amplitud de la educación superior hacia los grupos étnicos (pueblos originarios), la feminización de la matricula sigue en aumento y se están creando programas para estudiantes con discapacidad, el lento crecimiento al acceso del estudiante de menores ingresos económicos familiares (medido por quintiles de ingreso) se mantiene en el nuevo milenio, a diferencia, por ejemplo, de varios países latinoamericanos donde existen avances significativos en este sentido (Centro Interuniversitario de Desarrollo, 2016).

\section{MARCO TEÓRICO}

\section{REFERENCIAL}

2.1 Perfil del estudiante universitario global Los cambios en el perfil del estudiante de educación superior del nuevo milenio descritos en este documento se basaron primordialmente en literatura de Europa, América Latina y EEUU que destaca similitudes sociodemográficas, económicas y de circunstancias de vida de los estudiantes. Por ejemplo, en términos sociodemográficos, en la actualidad se encuentran estudiantes de todas las edades en los campus universitarios. Además, en su mayoría, los estudiantes representados son del sexo femenino y existe mayor diversidad de grupos "minoritarios" (indígenas, migrantes, estudiantes que padecen alguna discapacidad), como en el caso de países europeos. Por ejemplo, el Eurostudent, un estudio comparativo de las condiciones económicas y sociales del estudiante de educación superior europeo, define al estudiante no tradicional de la siguiente manera (Eurostudent 2018):

- Diferentes en relación a características socio-demográficas anteriores: Por ejemplo, se matriculan más estudiantes mayores a los 25 años y predominan las mujeres.

- Diferentes circunstancias de vida: estudiantes que tienen dependientes adicionales al de su cónyuge, los que son padres solteros, los que viven con alguna discapacidad.

- Diferentes características sociales: estudiantes sin padres con educación superior, estudiantes migrantes $\mathrm{y}$ estudiantes de entornos de bajo nivel socioeconómico.

- Otros indicadores que distinguen a este estudiante no tradicional incluyen: una entrada más tardía a la educación superior, estudiantes a medio-tiempo, trabajar a tiempo completo $\mathrm{y} / \mathrm{o}$ ser financieramente independiente de sus padres.

En lo concerniente a las características socioeconómicas de los estudiantes, la literatura analizada destaca, por ejemplo, un mayor acceso de estudiantes 
socioeconómicamente desaventajados/ bajos ingresos y el importante segmento que representan aquellos que trabajan, así como el crecimiento del estudiante de posgrado y de educación a distancia. Lo anterior, en el caso de América Latina, lo describió muy bien Rama (2007) en una investigación sobre las características de los nuevos estudiantes de la región. El autor destaca el aumento de la matrícula y la tasa de cobertura de la educación superior en la región debido, entre otros, al aumento en la urbanización, la transformación productiva, el cambio demográfico y la apertura económica de las sociedades latinoamericanas permitiendo a su vez una diversificación del perfil social del estudiante. Esta diversificación incluye la incorporación de estudiantes del interior de los países, estudiantes trabajadores, estudiantes a distancia, estudiantes multiétnicos y estudiantes con discapacidades. Otras de las principales razones para el aumento de la matrícula y diversificación del estudiantado incluyen el aumento de graduados de educación secundaria y un mayor acceso por parte de los dos quintiles de menores ingresos a nivel regional. (Ferreyra et al., 2017).

Estos nuevos perfiles también han causado el surgimiento de nuevos métodos de enseñanza y aprendizaje. Entre estos métodos destaca un crecimiento de la enseñanza a

* El muestreo implementado se denomina multietápico, es decir, consiste en una selección en primera instancia de las distancia y virtual (por ejemplo: cursos en línea para masas o Moocs, por sus siglas en inglés); así como la ampliación de estilos de aprendizaje facilitados por los avances tecnológicos y de la comunicación (Youtube, por ejemplo) (Selingo, 2018). Finalmente, la literatura destaca el futuro que debe afrontar el estudiante una vez graduado de su programa de estudios y recomienda facilitar $\mathrm{y}$ fortalecer sistemas de información laboral a través de datos sobre retornos laborales, demanda de empleo y otros para que el estudiante pueda tomar mejores decisiones una vez haya culminado sus estudios universitarios (Novella \& Rucci, 2018).

\section{METODOLOGÍA Y FUENTE DE} DATOS

La metodología utilizada se basó en una revisión bibliográfica de informes $y$ publicaciones de entidades universitarias $\mathrm{e}$ instituciones públicas y privadas de diferente naturaleza, además de la aplicación de una Encuesta denominada "Características de los Estudiantes de las IES de Honduras" a una muestrał de estudiantes de cinco IES representativas del Sistema de Educación Superior (SES) de Honduras, y la cual representó la principal fuente de información primaria de esta investigación.

IES o unidades primarias y después de los estudiantes o unidades secundarias. 
Las cinco IES fueron seleccionadas de acuerdo con la clasificación realizada por la Dirección de Educación Superior de su matrícula estudiantil: macro, grandes, medianas y pequeñas. De esta manera, forman parte de la muestra la Universidad Nacional Autónoma de Honduras (UNAH) y la Universidad Pedagógica Nacional Francisco Morazán (UPNFM), las dos IES públicas que tienen la mayor matrícula estudiantil, catalogadas como "macrouniversidad" y universidad "grande" , respectivamente. La Universidad Católica de Honduras (UNICAH) representó a la IES privada de tamaño "grande" y la Universidad Metropolitana de Honduras (UMH) fue la "mediana". La IES "pequeña" escogida fue el Centro de Diseño, Arquitectura y Construcción (CEDAC). Para el tamaño de la muestra de cada IES se tomó en cuenta un intervalo de confianza de $95 \%$ y un error del $5 \%$.

Los estudiantes fueron escogidos por autoridades de las IES de manera aleatoria con la condición de que pertenecieran a la matrícula de reingreso y fueran proporcionalmente representativos a la matrícula del área de conocimiento ofrecida por cada IES. Se consideraron estudiantes pertenecientes a la categoría 6 (grado en educación terciaria o nivel equivalente $\mathrm{y}$ pertenecientes a licenciaturas) de la Clasificación Internacional Normalizada de la Educación (CINE) de la Organización de las Naciones Unidas para la Educación, la Ciencia y la Cultura (UNESCO). El número total de estudiantes de modalidad presencial y de distancia por IES encuestados, entre los meses de agosto y diciembre del 2017, se muestra en la siguiente tabla:

Tabla 1. Número de Estudiantes por IES (modalidad presencial y distancia)

\begin{tabular}{|l|r|r|}
\hline \multicolumn{1}{|c|}{ IES } & Número de Estudiantes & \% de Representación \\
\hline CEDAC & 44 & 2.3 \\
\hline UMH & 118 & 6.1 \\
\hline UMH Distancia & 228 & 11.7 \\
\hline UNAH & 463 & 23.8 \\
\hline UNAH Distancia & 49 & 2.5 \\
\hline UNAH VS & 97 & 5.0 \\
\hline Unicah & 370 & 19.0 \\
\hline Unicah SPS & 172 & 8.9 \\
\hline UPNFM & 258 & 13.3 \\
\hline UPNFM Distancia & 144 & 7.4 \\
\hline Total & $\mathbf{1 9 4 3}$ & $\mathbf{1 0 0 . 0}$ \\
\hline
\end{tabular}

Fuente: Elaboración propia en base a muestra de cinco IES hondureñas 
En el caso del CEDAC, la UNAH y UNICAH los estudiantes de modalidad presencial representaron sus campus de Tegucigalpa y San Pedro Sula; los estudiantes de la modalidad de distancia de la UNAH tuvieron representación en los centros regionales de las ciudades de El Progreso, Tocoa y Danlí, así como en el campus principal de Tegucigalpa. La UPNFM aplico las encuestas a estudiantes presenciales predominantemente de Tegucigalpa, San Pedro Sula y la Ceiba. La mayoría de sus estudiantes a distancia representaron los campus de Tegucigalpa, La Ceiba y Comayagua. La UMH, presencial y distancia, concentró la representación de sus estudiantes en Tegucigalpa, Choluteca y La Esperanza, Intibucá. Los estudiantes a distancia encuestados sumaron un total de 410 , de los cuales casi un $80 \%$ representaron a la UPNFM.

Los estudiantes se segmentaron por las áreas de conocimiento según la Clasificación Internacional Normalizada de la Educación (CINE) de la UNESCO, quedando divididas de la siguiente manera:

- Administración de Empresas y Derecho

- Humanidades y Arte

- Servicios

- Ciencias Sociales, Periodismo e Información

- Educación
- Salud y Bienestar

- Ingeniería, Industria y Construcción,

- Ciencias Naturales, Matemáticas, Estadística y

- Tecnologías de la Información y Comunicación

El 27\% de los encuestados representaron el área de Administración de Empresas y Derecho, el 3\% el de Artes y Humanidades, el $12 \%$ el de ciencias sociales, periodismo e información, 25\% de Educación, el 2\% el de Ciencias Naturales, Matemáticas y Estadística, 12\% de Ingeniería, Industria y Construcción, $12 \%$ Salud y Bienestar y un $6 \%$ las TICs. Las áreas de Agricultura y de Servicios no fueron consideradas por su baja representatividad. En el caso del sector de Agricultura, la mayoría de sus estudiantes residen en los campus universitarios por lo que sería conveniente realizar un análisis aparte para este segmento estudiantil. Finalmente, se encuestó a una muestra de estudiantes de posgrado. Las encuestas se aplicaron específicamente a los estudiantes de maestría (nivel 7 del CINE) por representar estos una mayoría entre este segmento estudiantil. Se obtuvo una muestra de 318 estudiantes para esta población, en su mayoría de Tegucigalpa y San Pedro Sula. El total de encuestas digitales (presenciales, distancia y posgrado) fue de 2,261 representando aproximadamente el $1.1 \%$ del 
total de la matrícula del año 2016 de los estudiantes del sistema de educación superior hondureño.

Tabla 2. Estudiantes de Maestría por IES

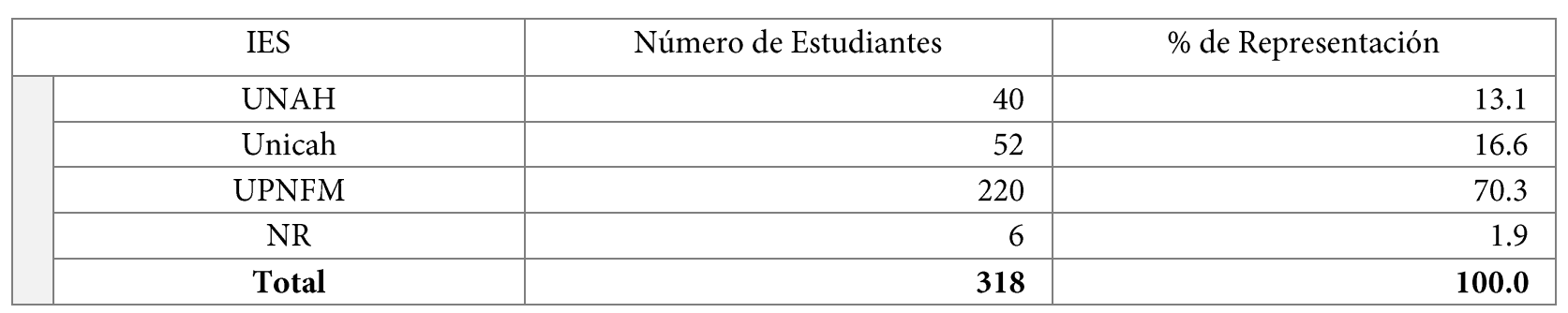

Fuente: Elaboración propia en base a muestra de tres IES hondureñas

\section{DISCUSIÓN DE RESULTADOS}

\subsection{Cambios en la matrícula de estudiantes de educación superior}

En Honduras ha existido un aumento progresivo en la matrícula de educación superior en el siglo XXI. La matrícula en educación superior en Honduras era de 33,188 estudiantes en 1982, teniendo el $60 \%$ una edad promedio entre los 19 y 25 años (Zuniga, 1987); posteriormente pasó a 144, 786 estudiantes en el 2006 y a 201,821 estudiantes en el 2016. Estos últimos representaron el $16.5 \%$ del total de los 1.22 millones de jóvenes hondureños entre los 18 y 24 años (DES, 2018).

La mayor cantidad de matriculados en las IES del sistema se encuentran actualmente entre los 20-24 años, constituyendo aproximadamente el $43 \%$ del total de los

\footnotetext{
$\S$ Tasa bruta de escolarización: El porcentaje de la población que, independientemente de su edad, participa en el nivel superior en relación con la cohorte en edad de cursar
}

estudiantes de educación superior. En segundo lugar, con un $23 \%$ están aquellos entre los 25 y 29 años (DES, 2018) (en EEUU aproximadamente el $62 \%$ de los estudiantes tienen menos de 25 años y el 38\% tiene más de 25 años (National Center for Education Statistics (2018)).

La tendencia hacia el aumento de la matrícula coincide también con la expansión de las universidades privadas que se dio con mayor fuerza en la primera década de este milenio (DES, 2018). Sin embargo, en cuanto a la masificación de la educación superior, Honduras muestra una tasa bruta de escolarización§ inferior al 30\% (Cinda, 2016); contrario al promedio regional latinoamericano y del caribe que creció de aproximadamente $22 \%$ en el 2000 a $46 \%$ en el 2015 (UNESCO, 2017).

estudios superiores. En Honduras fue de aproximadamente $22 \%$ para el año 2016 
En lo concerniente al sexo de los estudiantes de este nivel educativo, Honduras también forma parte del fenómeno de feminización. A principios de los 1980s ya se hacía referencia a este proceso, destacándose la participación de la mujer en las áreas de la educación, las ciencias sociales y las humanidades (Zuniga, 1987). El número de mujeres que en la actualidad se matriculan (57\%) y gradúan (65\%) de educación superior, ratifican su mayor representatividad (DES, 2018). Además, la tendencia de una mayor participación femenina se da en casi todas las áreas del conocimiento, siendo excepción la ingeniería, industria y construcción y las tecnologías de la información y la comunicación.

\subsection{Cambios sociodemográficos}

\subsubsection{Jornada de Estudios ${ }^{* *}$}

Las jornadas de estudio preferidas por el estudiante universitario hondureño son variadas. En la actualidad predominan la jornada mixta $\dagger \uparrow(31 \%)$ y la matutina $(25 \%)$. Una modalidad que parece estar creciendo de manera importante es la de estudios a distancia que alcanza el $21 \%$ y que se explicaría por las disposiciones de varias universidades a ofrecer dichos servicios. La incorporación de nuevas tecnologías en los últimos quince años (redes sociales, Youtube,

\footnotetext{
** A partir de esta sección, las tablas y gráficas presentadas en el documento son los resultados de la encuesta aplicada a
}

bibliotecas virtuales) seguramente incrementará el porcentaje de estudiantes que prefieran la modalidad de distancia.

Tabla 3. Jornada Actual de Estudios

\begin{tabular}{|l|r|r|}
\hline Matutina & 484 & $25 \%$ \\
\hline Vespertina & 273 & $14 \%$ \\
\hline Nocturna & 136 & $7 \%$ \\
\hline Mixta & 598 & $31 \%$ \\
\hline Semi-Presencial & 12 & $1 \%$ \\
\hline Distancia & 410 & $21 \%$ \\
\hline Virtual & 2 & $0 \%$ \\
\hline No Respondieron (NR) & 28 & $1 \%$ \\
\hline Total & $\mathbf{1 9 4 3}$ & $\mathbf{1 0 0 \%}$ \\
\hline
\end{tabular}

Fuente: Elaboración propia en base a Encuesta de "Características de los Estudiantes de las IES de Honduras"

\subsubsection{Estudiantes Provenientes de Grupos Étnicos}

Los estudiantes provenientes de los pueblos originarios también forman parte de la masificación de la educación superior, tal y como lo señalan López y Moncada (s.f.) al hacer mención del aumento significativo de estudiantes de origen indígena $y$ afrodescendiente en la UNAH, quienes pasaron de representar $2.9 \%$ en el 2012 a $9.1 \%$ en el 2015.

La información recolectada muestra que el $14 \%$ de la población estudiantil se identificó como parte de los grupos étnicos o pueblos originarios; los lencas son mayoritarios con

una muestra de estudiantes de cinco IES realizadas entre agosto y diciembre del 2017.

${ }^{\dagger}$ La jornada mixta se refiere a cualquier combinación de las jornadas regulares. 
un 9\%, mientras que los Maya-Chortí, Garífunas, Miskito y Tolupanes representan el 5\%. Un $71 \%$ se identificó como población mestiza (Figura 1). El acceso de grupos étnicos a la educación universitaria se ha visto favorecido, entre otras cosas, por el establecimiento de centros regionales y locales de las universidades tanto públicas como privadas.

Figura 1. Grupo Étnico al que dicen Pertenecer los Estudiantes (en porcentajes)

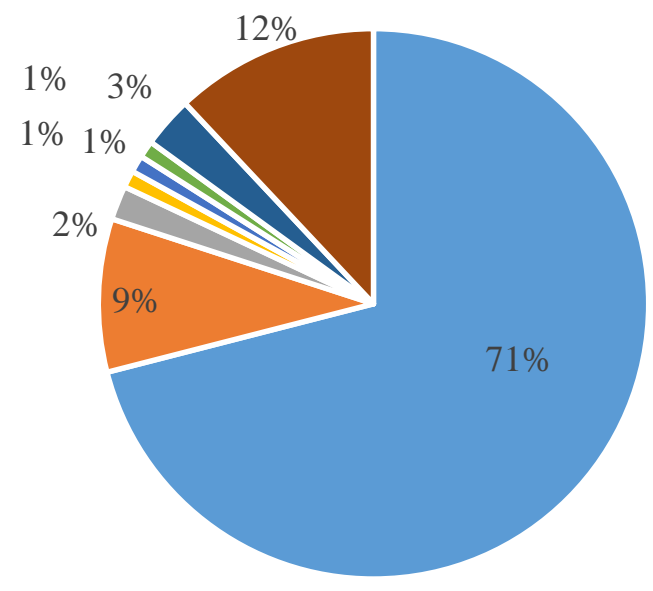

\author{
- Mestizo \\ - Lenca \\ - Maya-Chorti \\ - Garífuna \\ - Miskito \\ - Tolupan \\ - Otros/No lo Saben \\ - NR
}

Fuente: Elaboración propia en base a Encuesta de "Características de los Estudiantes de las IES de Honduras"

Estos datos sugieren un incremento en el acceso de los grupos étnicos en el caso de Honduras para este nuevo milenio, contrastando con un estancamiento en América Latina (Rama, 2007). Sin embargo, es importante continuar analizando la equidad en la permanencia y egreso de este segmento estudiantil como lo han realizado Lopez y Moncada (s.f.).

\subsection{Circunstancias de Vida}

\subsubsection{Estado Civil e hijos}

El estado civil de los estudiantes de pregrado es predominantemente de soltería (82\% expresaron tener este estado civil) mientras que solamente el $15 \%$ están casados o en unión libre (Figura 2). La mayoría de los estudiantes de pregrado (79\%) reportaron no tener hijos; $11 \%$ reportó tener uno, y los que tienen 2 o 3 hijos suman 7\% (Tabla 4). Lo anterior tiende a mostrar que el estudiante hondureño se encuentra en transición hacia lo que se califica como "no tradicional": de mayor edad, con hijos, etc., pero sin alcanzar el nivel de otras regiones como Estados Unidos y Europa donde los datos demuestran que el incremento de cobertura en la educación superior se debe en buena parte a estudiantes mayores de 25 años que trabajan y tienen hijos (Eurostudent, 2018). 
Figura 2. Estado Civil de los estudiantes de educación superior en Honduras (en porcentajes)

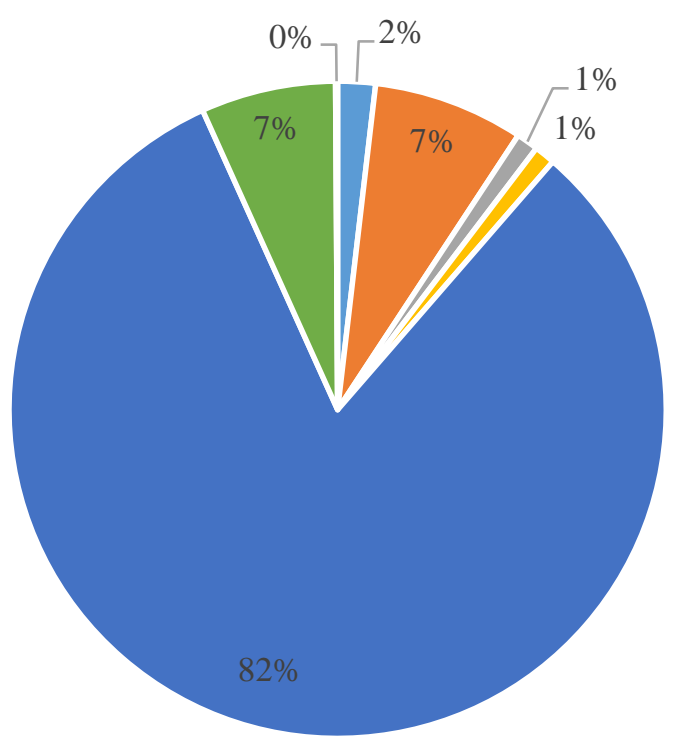

- NR

- Casado(a)

- Divorciado(a)

- Separado(a)

- Soltero(a)

- Unión Libre

- Viudo(a)

Fuente: Elaboración propia en base a Encuesta de "Características de los Estudiantes de las IES de Honduras"

Tabla 4. Número de Hijos de Estudiantes de Pregrado

\begin{tabular}{|c|c|c|}
\hline Núm. de Hijos & Núm. de Estudiantes & \% de Representatividad \\
\hline $\mathbf{0}$ & 1,526 & $79 \%$ \\
\hline $\mathbf{1}$ & 214 & $11 \%$ \\
\hline $\mathbf{2}$ & 104 & $5 \%$ \\
\hline $\mathbf{3}$ & 31 & $2 \%$ \\
\hline $\mathbf{4}$ & 3 & $0 \%$ \\
\hline Más de 4 & 2 & $0 \%$ \\
\hline NR & 63 & $3 \%$ \\
\hline Total & $\mathbf{1 , 9 4 3}$ & $\mathbf{1 0 0 \%}$ \\
\hline
\end{tabular}

Fuente: Elaboración propia en base a Encuesta de "Características de los Estudiantes de las IES de Honduras”

\subsubsection{Residencia y Financiamiento de Gastos}

La mayor parte de los estudiantes (54\%) comparten residencia con su padre o madre, el $18 \%$ con otros familiares y el $13 \%$ con su cónyuge y/o hijos (Figura 3).
Los estudiantes universitarios financian los gastos de su entorno económico universitario (alojamiento, comida, transporte, materiales de estudio), de la siguiente manera: el 62\% proceden de recursos proporcionados por 
padre/madre/otros familiares, un $27 \%$ son financiados a través de los ingresos propios por trabajo y solamente $4 \%$ son becados. En contraste, en Europa aproximadamente el $14 \%$ de los estudiantes recibe fondos públicos a través de becas o préstamos (Eurostudent, 2018) y en América Latina el acceso a la educación superior en el nuevo milenio ha crecido en parte por los préstamos estudiantiles y becas, en particular a los quintiles de menores ingresos $y$ especialmente en el sector privado. (Ferreyra et. al., 2017).

La figura 4 muestra las principales fuentes de financiamiento para el estudiante de educación superior hondureño. Este y el acápite anterior parecen corroborar la transición lenta hacia el estudiante "no tradicional" que se da en Honduras.

Figura 3. Con quien viven los estudiantes (en porcentajes)

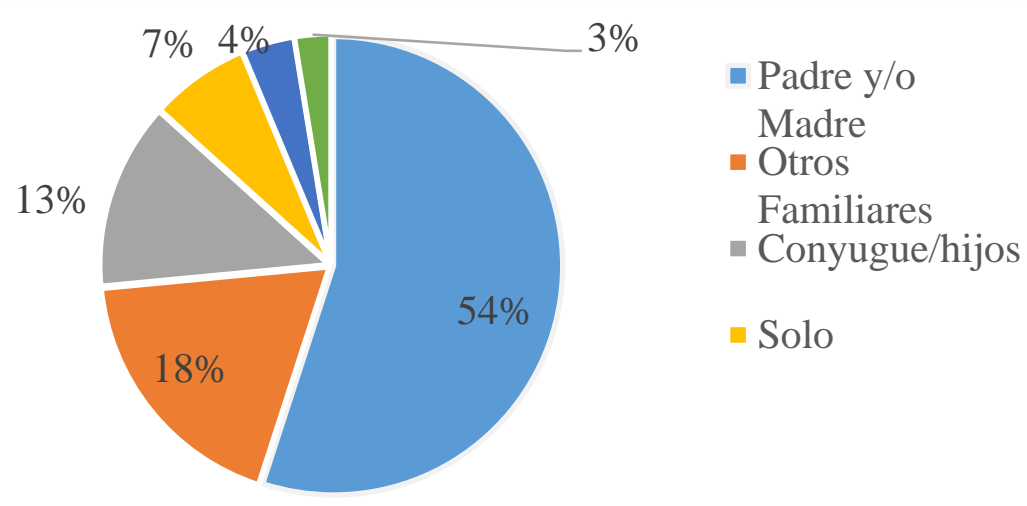

Fuente: Elaboración propia en base a Encuesta de "Características de los Estudiantes de las IES de Honduras"

Figura 4. Procedencia de gastos de alojamiento, comida, transporte, materiales de estudio

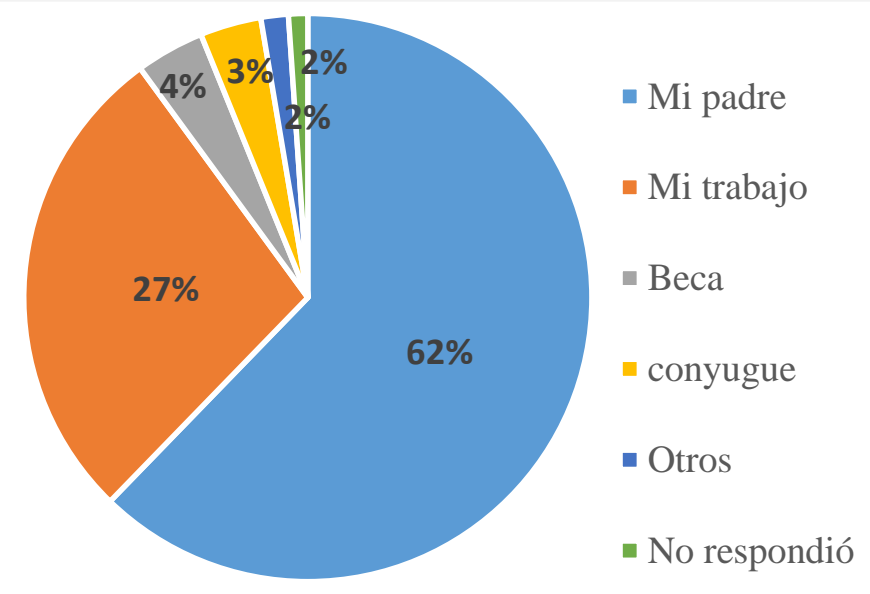

Fuente: Elaboración propia en base a Encuesta de "Características de los Estudiantes de las IES de Honduras" 


\subsubsection{Estudiantes con Discapacidad}

Se calcula que en el país existen aproximadamente 800,000 mil personas con discapacidad, es decir, un estimado del $10 \%$ de la población tiene algún problema de discapacidad (INE, 2016). Según el Informe de Educación Superior en Iberoamérica (2016), existen acciones en algunas IES hondureñas, mayoritariamente públicas, donde se han realizado programas para estudiantes discapacitados, considerando adaptaciones, programas especializados, equipos técnicos y voluntarios que apoyen a personas con discapacidad.

La información recolectada muestra que solo 27 estudiantes de pregrado reportaron tener una discapacidad de un total de 1943, es decir, aproximadamente el 1.4\%.

Tabla 5. Estudiantes con discapacidad

\begin{tabular}{|l|r|r|}
\hline $\begin{array}{c}\text { ¿Sufre usted de algún tipo } \\
\text { de discapacidad física, } \\
\text { mental o de alguna otra } \\
\text { naturaleza? }\end{array}$ & $\begin{array}{c}\text { Núm. de } \\
\text { Estudiant } \\
\text { es }\end{array}$ & $\begin{array}{r}\text { Porcentaje de } \\
\text { Participación }\end{array}$ \\
\hline Si & 27 & $1.4 \%$ \\
\hline No & 1788 & $92 \%$ \\
\hline NR & 128 & $6.6 \%$ \\
\hline Total & $\mathbf{1 9 4 3}$ & $\mathbf{1 0 0 \%}$ \\
\hline
\end{tabular}

Fuente: Elaboración propia en base a Encuesta de "Características de los Estudiantes de las IES de Honduras"

\#‡ Este porcentaje es menor al 71\% que reporta el registro de admisión de la UNAH para el examen de admisión de esta IES.

\subsection{Otras Características Socioeconómicas \\ 4.4.1 Estudiantes que Trabajan}

El estudiante de pregrado hondureño en su mayoría no trabaja (59\%). 㭏 En el caso de los estudiantes que trabajan, las condiciones en Honduras son similares a las de otros países latinoamericanos; es decir, hay una cantidad significativa de jóvenes que trabajan y que asisten a un centro de educación superior en las noches o antes de su jornada laboral. Los estudiantes de pregrado trabajan en las siguientes jornadas: a tiempo completo (23\%), a medio tiempo (9\%), otro tipo de jornada o en los recesos académicos (3\%). Ver figura 5.

El salario mínimo promedio en Honduras fue de alrededor de 8,450 lempiras en el 2017\$\$; la mayoría (62\%) de los estudiantes universitarios que dijeron trabajar obtuvo una remuneración por debajo de ese monto y solamente el 36\% tuvo salarios mensuales por encima de 10,000 lempiras. ${ }^{\star * *}$ Estos niveles bajos de salarios (23\% recibieron menos de la mitad del salario mínimo) muestran en alguna forma la situación difícil del mercado laboral en Honduras.

\footnotetext{
$\$$ Alrededor de US359 al tipo de cambio de finales de 2017.

*** Alrededor del US $\$ 424$ al tipo de cambio de finales de 2017.
} 
Figura 5. Situación laboral de los estudiantes (en porcentajes)

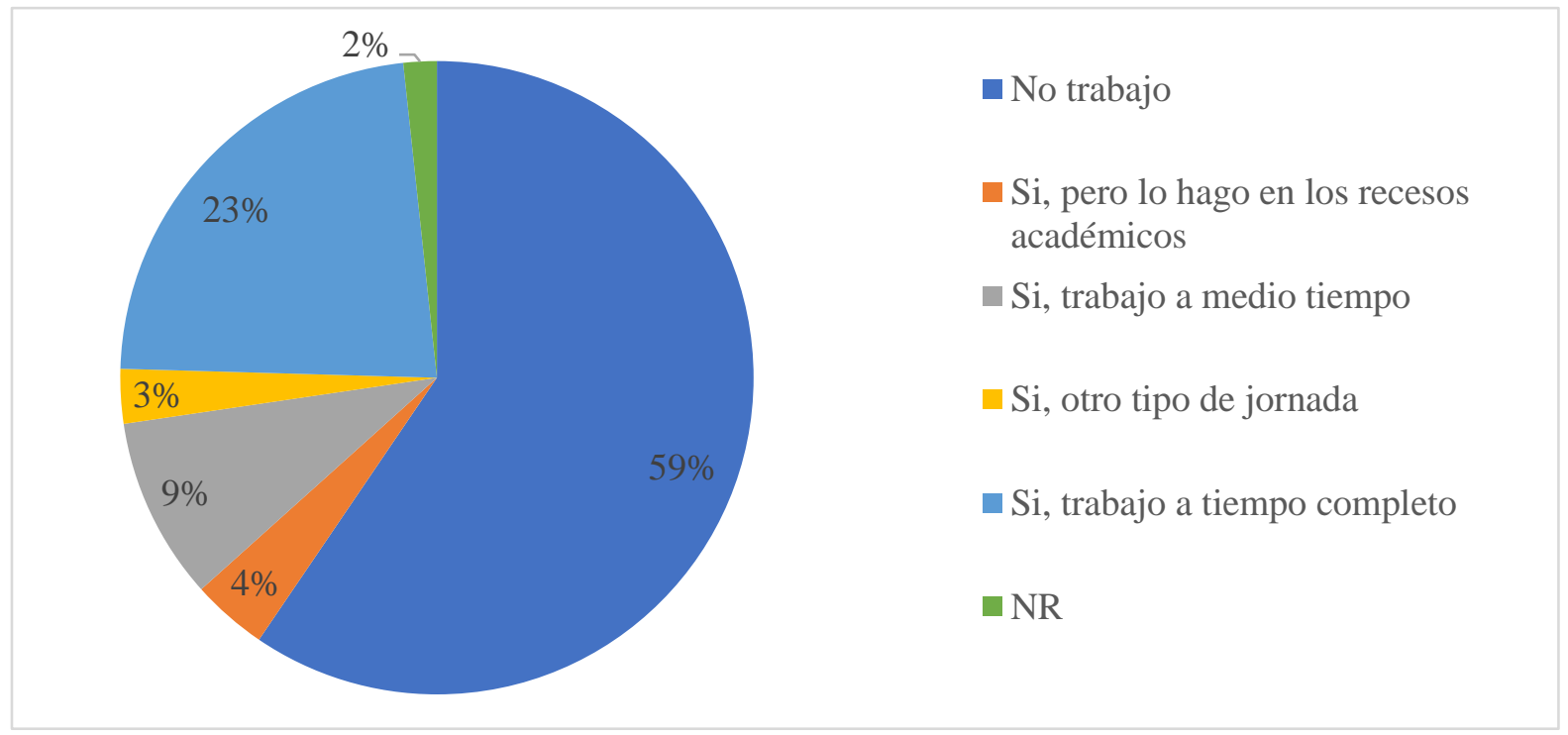

Fuente: Elaboración propia en base a Encuesta de "Características de los Estudiantes de las IES de Honduras"

Figura 6. Salario promedio mensual de los estudiantes que trabajan

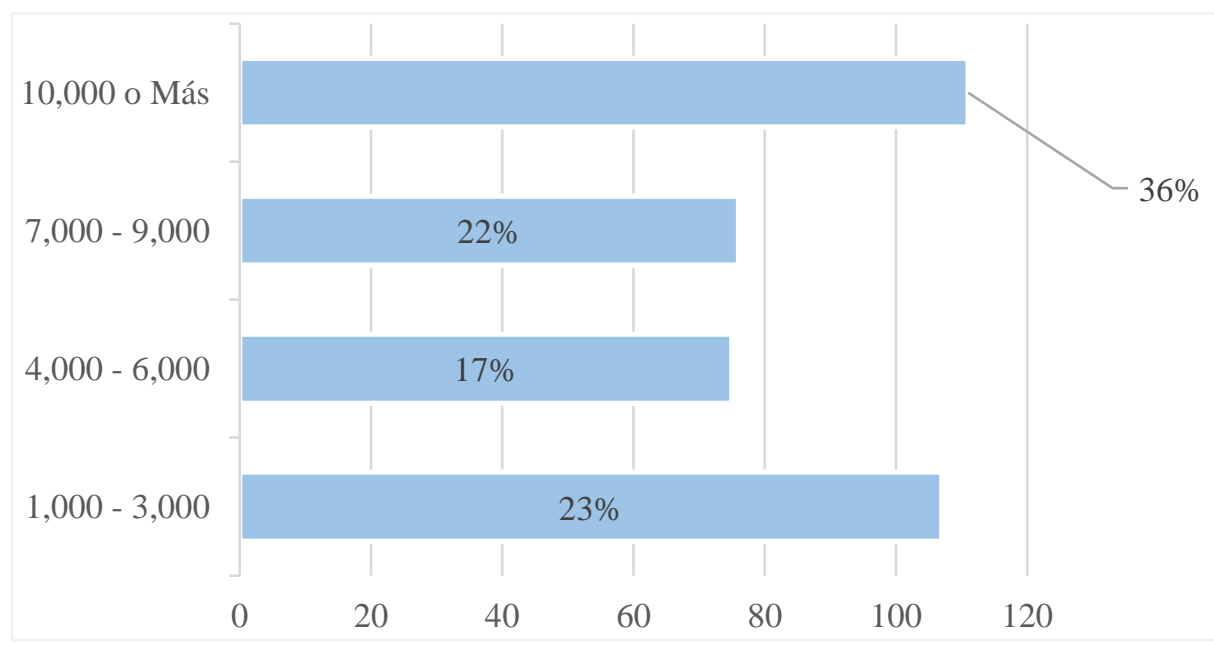

Fuente: Elaboración propia en base a Encuesta de "Características de los Estudiantes de las IES de Honduras"

\subsubsection{Nivel Educativo de los Padres}

Los estudiantes también fueron consultados sobre la educación de su padre y de su madre. Casi un 36\% de los estudiantes reporto tener padres con un nivel educativo que supera el nivel educativo medio. Este porcentaje supera ampliamente el 7\% del total de jefes de hogar hondureños con un nivel educativo superior (INE, 2017). Los valores son muy similares 
para el caso de la madre, registrando incluso un valor ligeramente mayor para las que tienen un nivel superior universitario. Estos datos coinciden con las tendencias internacionales que explican que existe un mayor acceso para el estudiante de educación superior cuyos padres se graduaron de este nivel educativo.

\subsubsection{Ingresos familiares}

El 64\% de los estudiantes tienen ingresos mensuales familiares menores a los 20,000 mil lempiras ${ }^{\dagger \dagger}$; este último ingreso representaría aproximadamente dos veces y medio el salario mínimo en Honduras para 2017 y estaría mostrando un nivel de acceso mayor a familias de menores ingresos del que ha sido tradicional, aunque la desigualdad se mantiene. Solamente un $8 \%$ de los estudiantes tienen ingresos familiares por arriba de los 50,000 lempiras, mientras que cerca de la cuarta parte está entre 20,000 y 50,000 lempiras (Tabla 6). Destaca en el cuadro anterior que el mayor porcentaje de estudiantes (27\%) tenga ingresos mensuales familiares en el rango entre los 5,000 y 10,000 mil lempiras; esto coincide con el ingreso de la mayor parte de los aspirantes al examen de admisión de la UNAH (la IES con el $44 \%$ de la matrícula de estudiantes del país) en el periodo de aplicación entre el 2006 y el 2014 (Sistema de Admisión, UNAH, 2014).
Tabla 6. Ingresos Familiares Mensuales (en lempiras)

\begin{tabular}{|l|r|r|}
\hline \multicolumn{1}{|c|}{ Ingreso } & Estudiantes & $\begin{array}{r}\text { Porcentaje de } \\
\text { Representación }\end{array}$ \\
\hline Menor a 5,000 & 245 & $13 \%$ \\
\hline 5,000 a 10,000 & 534 & $27 \%$ \\
\hline 10,001 a 20,000 & 465 & $24 \%$ \\
\hline 20,001 a 30,000 & 297 & $15 \%$ \\
\hline 30,001 a 50,000 & 214 & $11 \%$ \\
\hline 50,001 a 75,000 & 91 & $5 \%$ \\
\hline Mayor a 75,000 & 60 & $3 \%$ \\
\hline NR & 37 & $2 \%$ \\
\hline Total & $\mathbf{1 9 4 3}$ & $\mathbf{1 0 0} \%$ \\
\hline
\end{tabular}

Fuente: Elaboración propia en base a Encuesta de

"Características de los Estudiantes de las IES de Honduras"

\subsection{Estudiantes a Distancia y de Posgrado}

\subsubsection{Estudiantes a distancia}

En Honduras, la modalidad de educación a distancia representa alrededor de un $25 \%$ de la población estudiantil total. Las preguntas realizadas a este segmento estudiantil fueron las mismas que a los estudiantes de pregrado, encontrándose algunas diferencias significativas en los resultados. Por ejemplo, al realizar la pregunta sobre jornada de estudios y número de hijos, el 52\% del total de los estudiantes que reportaron tener uno o más hijos estudian bajo la modalidad de distancia, en comparación con el 15\% para el caso del estudiante con jornada matutina y el $14 \%$ con jornada mixta, tal y como se puede apreciar en la siguiente tabla:

\footnotetext{
${ }^{\dagger \dagger \dagger}$ Alrededor de US\$850 a finales de 2017.
} 
Tabla 7. Jornada de estudio y cantidad de hijos de los estudiantes

\begin{tabular}{|c|c|c|c|c|c|c|c|c|}
\hline \multirow{2}{*}{\multicolumn{2}{|c|}{ Descripción }} & \multicolumn{7}{|c|}{ ¿Tiene hijos/as? [Cantidad ] } \\
\hline & & \multirow{2}{*}{$\begin{array}{l}1 \\
103\end{array}$} & \multirow{2}{*}{$\begin{array}{l}2 \\
56 \\
\end{array}$} & \multirow{2}{*}{$\begin{array}{l}3 \\
22 \\
\end{array}$} & \multirow{2}{*}{$\begin{array}{l}4 \\
2\end{array}$} & \multirow{2}{*}{$\begin{array}{r}\text { más de } 4 \\
0 \\
\end{array}$} & \multirow{2}{*}{$\begin{array}{r}\text { Total } \\
183\end{array}$} & \multirow{2}{*}{$\begin{array}{r}\text { \% de } \\
\text { Representación } \\
52 \% \\
\end{array}$} \\
\hline \multirow{9}{*}{$\begin{array}{l}\text { Jornada } \\
\text { de } \\
\text { Estudios }\end{array}$} & Distancia & & & & & & & \\
\hline & Matutina & 31 & 18 & 3 & 0 & 0 & 52 & $15 \%$ \\
\hline & Mixta & 35 & 11 & 1 & 0 & 1 & 48 & $14 \%$ \\
\hline & Nocturna & 18 & 10 & 3 & 0 & 0 & 31 & $9 \%$ \\
\hline & Semi-Presencial & 3 & 2 & 0 & 0 & 0 & 5 & $1 \%$ \\
\hline & Vespertina & 24 & 7 & 1 & 0 & 0 & 32 & $9 \%$ \\
\hline & Virtual & 0 & 0 & 1 & 0 & 0 & 1 & $0 \%$ \\
\hline & $\mathrm{NR}$ & 0 & 0 & 0 & 1 & 1 & 2 & $1 \%$ \\
\hline & Total & 214 & 104 & 31 & 3 & 2 & 354 & $100 \%$ \\
\hline
\end{tabular}

Fuente: Elaboración propia en base a Encuesta de "Características de los Estudiantes de las IES de Honduras"

En relación con su situación laboral y, tomando en cuenta las tres jornadas de estudio de mayor popularidad, casi el 79\% de los estudiantes a distancia respondió tener alguna forma de trabajo, comparado con el $27 \%$ de los de jornada matutina y $22 \%$ de jornada mixta. Lo anterior refuerza la percepción de que los estudiantes que trabajan tienen familia, y reciben menos ingresos (como se verá a continuación) buscan formas alternativas para continuar sus estudios.

Tabla 8. Jornada de estudios y estudiantes que trabajan

\begin{tabular}{|l|r|r|r|}
\hline $\begin{array}{c}\text { Jornada } \\
\text { de } \\
\text { Estudio }\end{array}$ & $\begin{array}{c}\text { Número } \\
\text { total de } \\
\text { Estudiantes }\end{array}$ & $\begin{array}{c}\text { Estudiantes } \\
\text { que Trabajan }\end{array}$ & $\begin{array}{c}\% \\
\text { Estudiantes } \\
\text { trabajadores }\end{array}$ \\
\hline Distancia & 410 & 322 & $79 \%$ \\
\hline Matutina & 484 & 130 & $27 \%$ \\
\hline Mixta & 598 & 129 & $22 \%$ \\
\hline Totales & $\mathbf{1 4 9 2}$ & $\mathbf{5 8 1}$ & $\mathbf{3 9 \%}$ \\
\hline
\end{tabular}

Fuente: Elaboración propia en base a Encuesta de

“Características de los Estudiantes de las IES de Honduras”

Asimismo, se observa que los estudiantes de modalidad a distancia en un $81 \%$ reportaron tener ingresos familiares menores a los 20,000 mil lempiras, representando la jornada de estudios donde los estudiantes reportaron tener menores ingresos. La relación entre las principales jornadas de estudio y el ingreso mensual familiar es la siguiente: 
Tabla 9. Jornada de estudio e ingreso mensual de la familia

\begin{tabular}{|c|c|c|c|c|c|c|c|}
\hline \multirow{2}{*}{$\begin{array}{c}\text { Ingresos } \\
\text { Mensuales }\end{array}$} & Distancia & Matutina & Mixta & Nocturna & NR & \multirow[b]{2}{*}{ Total } & \multirow[b]{2}{*}{$\begin{array}{c}\% \text { de } \\
\text { Representación }\end{array}$} \\
\hline & $\begin{array}{c}\text { N. de } \\
\text { Estudiantes }\end{array}$ & $\begin{array}{c}\text { N. de } \\
\text { Estudiantes }\end{array}$ & $\begin{array}{l}\text { N. de } \\
\text { Estudi } \\
\text { antes }\end{array}$ & $\begin{array}{c}\text { N. de } \\
\text { Estudiant } \\
\text { es }\end{array}$ & $\begin{array}{c}\text { N. de } \\
\text { Estudiantes }\end{array}$ & & \\
\hline $\begin{array}{c}\text { Menor a } \\
5,000\end{array}$ & 73 & 60 & 60 & 11 & 0 & 204 & $12 \%$ \\
\hline $\begin{array}{l}5,001- \\
10,000\end{array}$ & 138 & 119 & 144 & 46 & 4 & 451 & $27 \%$ \\
\hline $\begin{array}{l}10,001- \\
20,000\end{array}$ & 117 & 126 & 129 & 27 & 0 & 399 & $24 \%$ \\
\hline $\begin{array}{c}20.001- \\
30,000\end{array}$ & 45 & 86 & 99 & 25 & 0 & 255 & $15 \%$ \\
\hline $\begin{array}{c}30,001- \\
50,000\end{array}$ & 20 & 50 & 92 & 18 & 0 & 180 & $11 \%$ \\
\hline $\begin{array}{l}50,001- \\
75,000\end{array}$ & 9 & 22 & 45 & 4 & 0 & 80 & $5 \%$ \\
\hline $\begin{array}{c}\text { mayor a } \\
75,000\end{array}$ & 5 & 18 & 25 & 3 & 1 & 52 & $3 \%$ \\
\hline NR & 3 & 3 & 4 & 2 & 23 & 35 & $2 \%$ \\
\hline Total & 410 & 484 & 598 & 136 & 28 & 1,656 & $100 \%$ \\
\hline
\end{tabular}

Fuente: Elaboración propia en base a Encuesta de "Características de los Estudiantes de las IES de Honduras"

\subsubsection{Estudiantes de Posgrado}

El promedio de edad de los estudiantes de maestría es de 32.5 años (muy cercano a los 32 años del promedio de edad del estudiante de posgrado de EEUU según el U.S. Department of Education (2018)). Es decir, siete años mayor que el promedio del estudiante de pregrado. El $60 \%$ de los estudiantes es del sexo femenino y al igual que el estudiante de pregrado, la mayoría dijeron estar solteros, pero contrario a los primeros quienes lo están en un $80 \%$, en el caso del estudiante de maestría es del 53\%. La tasa de casados y aquellos en unión libre sube a un $31 \%$ y $9 \%$ respectivamente, en comparación al 7\% de los estudiantes de pregrado para ambos casos; aumentando por 
consiguiente también las tasas de divorciados y separados de los estudiantes de maestría.

Tabla 10. Estado Civil del Estudiante de posgrado

\begin{tabular}{|l|r|r|}
\hline Estado Civil & $\begin{array}{c}\text { Número de } \\
\text { estudiantes }\end{array}$ & $\begin{array}{c}\text { Porcentaje de } \\
\text { participación }\end{array}$ \\
\hline Soltero & 169 & $53 \%$ \\
\hline Casado & 98 & $31 \%$ \\
\hline Divorciado & 5 & $2 \%$ \\
\hline Separado & 6 & $2 \%$ \\
\hline Unión libre & 29 & $9 \%$ \\
\hline NR & 11 & $4 \%$ \\
\hline Total & 318 & $100 \%$ \\
\hline
\end{tabular}

Fuente: Elaboración propia en base a Encuesta de

"Características de los Estudiantes de las IES de Honduras"

A diferencia del estudiante de pregrado, que solamente en un 18\% tiene hijos, más del $40 \%$ de los estudiantes de posgrado si reportan tenerlos, tal y como se aprecia en la siguiente tabla:

Tabla 11. Hijos de estudiantes de posgrado

\begin{tabular}{|l|r|r|}
\hline \multicolumn{1}{|c|}{ Hijos } & $\begin{array}{c}\text { Número de } \\
\text { estudiantes }\end{array}$ & $\begin{array}{c}\text { Porcentaje de } \\
\text { participación }\end{array}$ \\
\hline No tengo hijos & 169 & $53 \%$ \\
\hline 1 hijo & 66 & $21 \%$ \\
\hline 2 hijos & 38 & $12 \%$ \\
\hline 3 hijos & 27 & $8 \%$ \\
\hline 4 hijos & 6 & $2 \%$ \\
\hline NR & 12 & $4 \%$ \\
\hline Total & 318 & $100 \%$ \\
\hline
\end{tabular}

Fuente: Elaboración propia en base a Encuesta de "Características de los Estudiantes de las IES de Honduras" Ante la pregunta, ¿con quién vive actualmente?, el porcentaje de respuestas de los que dijeron vivir con su cónyuge/hijos o padre/madre fue de un $37 \%$ en ambos casos, contrario al $13 \%$ y $54 \%$, respectivamente, reportados por los estudiantes de pregrado. Las diferencias en los resultados entre los estudiantes de pregrado y posgrado mencionados anteriormente se deben seguramente a la transición en la vida de la mayoría de estos estudiantes.

\subsubsection{Estudiantes Trabajadores}

Una de las mayores diferencias entre los resultados de la encuesta de pregrado y posgrado, se da entre aquellos que trabajan a tiempo completo comparada con aquellos que no lo hacen.

El 70\% de los estudiantes de maestría reportó estar trabajando a tiempo completo, comparado con solo el $22 \%$ de los estudiantes de pregrado.

Tabla 12. Estudiantes de postgrado que trabajan

\begin{tabular}{|l|r|r|}
\hline \multicolumn{1}{|c|}{$\begin{array}{c}\text { Además de Estudiar, } \\
\text { Trabaja Actualmente }\end{array}$} & $\begin{array}{r}\text { Número de } \\
\text { estudiantes }\end{array}$ & $\begin{array}{r}\text { Porcentaje de } \\
\text { participación }\end{array}$ \\
\hline No trabajo & 15 & $5 \%$ \\
\hline Si, otro tipo de trabajo & 11 & $3 \%$ \\
\hline $\begin{array}{l}\text { Si, pero lo hago en los } \\
\text { recesos académicos }\end{array}$ & 1 & $0 \%$ \\
\hline $\begin{array}{l}\text { Si, trabajo a medio } \\
\text { tiempo }\end{array}$ & 57 & $18 \%$ \\
\hline $\begin{array}{l}\text { Si, trabajo a tiempo } \\
\text { completo }\end{array}$ & 224 & $70 \%$ \\
\hline NR & 10 & $3 \%$ \\
\hline Total & 318 & $100 \%$ \\
\hline
\end{tabular}

Fuente: Elaboración propia en base a Encuesta de "Características de los Estudiantes de las IES de Honduras"

El ingreso mensual familiar del estudiante de posgrado también representa una variable importante. En la siguiente tabla se observa 
que los ingresos mensuales familiares mejoran en el caso del estudiante de posgrado. Por ejemplo, el porcentaje de estudiantes con ingresos mensuales familiares de menos de 20 mil lempiras es de aproximadamente un $43 \%$, contrario al $64 \%$ reportado por el estudiante de pregrado. Entre los 20mil y 50mil lempiras se encuentran los ingresos familiares de un $45 \%$ de los estudiantes de maestría, contrario al $26 \%$ reportado por los estudiantes de pregrado.

Tabla 13. Ingreso estimado mensual de la familia del estudiante de postgrado (en lempiras)

\begin{tabular}{|c|c|c|}
\hline Ingreso & $\begin{array}{l}\text { Número de } \\
\text { Estudiantes }\end{array}$ & $\begin{array}{l}\text { Porcentaje de } \\
\text { Participación }\end{array}$ \\
\hline Menor a 5,000 & 12 & $4 \%$ \\
\hline $5,000-10,000$ & 41 & $13 \%$ \\
\hline $10,001-20,000$ & 85 & $27 \%$ \\
\hline $20,001-30,000$ & 84 & $26 \%$ \\
\hline $30,001-50,000$ & 59 & $19 \%$ \\
\hline $50,001-75,000$ & 15 & $5 \%$ \\
\hline Mayor a 75,000 & 10 & $3 \%$ \\
\hline NR & 12 & $4 \%$ \\
\hline Total & 318 & $100 \%$ \\
\hline
\end{tabular}

Fuente: Elaboración propia en base a Encuesta de “Características de los Estudiantes de las IES de Honduras"

\subsection{Expectativas Hacia el Futuro}

Existe un creciente número de publicaciones sobre las IES del futuro y los conocimientos que se esperan de los estudiantes de estos centros una vez se encuentren en el mundo laboral. Varias mencionan la renovación continua del conocimiento o aprendizaje para toda la vida, así como el auge de la enseñanza de cursos y carreras virtuales. Es por lo que se incluyeron al final de la encuesta tres preguntas sobre las expectativas al futuro que tiene el estudiante. La primera de ellas le preguntaba al estudiante que evaluará cuales eran sus posibilidades en el mercado laboral nacional e internacional, una vez graduado de su programa de estudios actual. En el caso de las opciones de "buenas", el optimismo existente para encontrar un trabajo en el mercado laboral del país como en el extranjero es similar. Sin embargo, se reducen las respuestas en el caso de la opción de "muy buenas". En el caso de las opciones "muy malas" y "malas", existe un mayor pesimismo de encontrar un trabajo a nivel nacional que en el extranjero, como se observa en la siguiente tabla: 
Tabla 14. Posibilidades en el mercado laboral

\begin{tabular}{|c|c|c|c|c|c|c|c|c|}
\hline \multirow{3}{*}{ Descripción } & \multicolumn{8}{|c|}{$\begin{array}{c}\text { ¿Cómo evalúa sus posibilidades en el mercado laboral una vez graduado de su actual programa de } \\
\text { estudios? }\end{array}$} \\
\hline & \multicolumn{2}{|l|}{ Buenas } & \multicolumn{2}{|c|}{ Muy buenas } & \multicolumn{2}{|l|}{ Malas } & \multicolumn{2}{|c|}{ Muy malas } \\
\hline & $\begin{array}{l}\text { Núm. de } \\
\text { Estudiantes }\end{array}$ & $\%$ & $\begin{array}{l}\text { Núm. de } \\
\text { Estudiant } \\
\quad \text { es }\end{array}$ & $\%$ & $\begin{array}{l}\text { Núm. de } \\
\text { Estudiantes }\end{array}$ & $\%$ & $\begin{array}{l}\text { Núm. } \\
\text { de } \\
\text { Estudia } \\
\text { ntes }\end{array}$ & $\%$ \\
\hline $\begin{array}{l}\text { En el } \\
\text { extranjero }\end{array}$ & 506 & $26 \%$ & 614 & $32 \%$ & 153 & $8 \%$ & 163 & $8 \%$ \\
\hline En el país & 901 & $46 \%$ & 425 & $22 \%$ & 459 & $24 \%$ & 421 & $22 \%$ \\
\hline NR/Otras & 536 & $28 \%$ & 904 & $47 \%$ & 1331 & $69 \%$ & 1359 & $70 \%$ \\
\hline Total & 1943 & $100 \%$ & 1943 & $100 \%$ & 1943 & $100 \%$ & 1943 & $100 \%$ \\
\hline
\end{tabular}

Fuente: Elaboración propia en base a Encuesta de "Características de los Estudiantes de las IES de Honduras"

En relación con su futuro, por último, se les consultó a los estudiantes sobre sus planes un año después de concluidos sus estudios. Un $44 \%$ respondió que buscará un trabajo al finalizar sus estudios, un $27 \%$ dijo que continuará estudiando, y un $11 \%$ dijo que establecer su propia empresa era su principal prioridad.

Tabla 15. Planes de los estudiantes al finalizar su carrera

\begin{tabular}{|l|r|r|}
\hline \multicolumn{1}{|c|}{$\begin{array}{c}\text { Al finalizar su actual } \\
\text { programa de estudios, } \\
\text { qué planes tiene para el } \\
\text { año siguiente }\end{array}$} & $\begin{array}{c}\text { Número de } \\
\text { Estudiantes }\end{array}$ & $\begin{array}{c}\text { Porcentaje } \\
\text { de } \\
\text { participació } \\
\text { n }\end{array}$ \\
\hline $\begin{array}{l}\text { Buscar empleo } \\
\text { Continuar con mi } \\
\text { empleo actual }\end{array}$ & 851 & $44 \%$ \\
\hline $\begin{array}{l}\text { Continuar } \\
\text { estudiando }\end{array}$ & 112 & $6 \%$ \\
\hline $\begin{array}{l}\text { Establecer mi propia } \\
\text { empresa }\end{array}$ & 215 & $11 \%$ \\
\hline $\begin{array}{l}\text { Emigrar del país y } \\
\text { buscar empleo en el } \\
\text { extranjero }\end{array}$ & 105 & $5 \%$ \\
\hline Aún no lo sé & 29 & $1 \%$ \\
\hline NR & 1943 & $100 \%$ \\
\hline Total & & $6 \%$ \\
\hline
\end{tabular}

\section{CONCLUSIONES}

El perfil socio-demográfico y económico del estudiante universitario hondureño de la actualidad presenta tendencias similares a las observadas a nivel global; no obstante, estas tendencias son menos marcadas en el tiempo por factores vinculados a problemas estructurales del país, entre ellos: la baja cobertura de la educación secundaria, la fuerte desigualdad en la distribución del ingreso, el desempleo y la pobreza. A continuación, se resumen algunas de las principales características del estudiante de educación superior hondureño del nuevo milenio:

\section{Sociodemográficas:}

- Un aumento de la tasa de cobertura de aproximadamente 9\% $\quad(33,000$ estudiantes, Zuniga, 1987) a un $22 \%$, (más de 201,800 estudiantes, DES, 2016). Sin embargo, esta cobertura se 
mantiene debajo del promedio latinoamericano de $44 \%$.

- Este incremento en cobertura y diversificación se ha representado, entre otros, con una mayor presencia de mujeres y estudiantes de grupos étnicos/pueblos originarios. Además, ha sido tanto en sectores urbanos como rurales debido en buena parte al aumento de centros universitarios regionales (especialmente de carácter privado).

- Es importante mencionar que una mayor presencia de estudiantes de grupos étnicos o pueblos originarios no equivale a equidad en la permanencia y el egreso y un mayor número de mujeres no representa mejores salarios que el de los hombres en el mundo laboral.

- La modalidad de estudio a distancia también ha ascendido notablemente y se acercan a un cuarto del total de los estudiantes universitarios.

- La edad del estudiante de educación superior hondureño de pregrado se mantiene similar a lo observado a principios de los 1980s, es decir en un rango entre los 19 y 25 años. Por lo tanto, no se ve un crecimiento en la matricula del estudiante "no tradicional" tan evidente como en otros países donde existe una representación importante de estudiantes que son mayores de 25 años.

\section{Socioeconómicas:}

- La mayoría de los estudiantes de pregrado que trabajan tienen ingresos menores a los 10,000 mil lempiras. En el caso de los ingresos familiares, el $64 \%$ de los estudiantes reporto tener menores a los 20 mil lempiras.

- La literatura atribuye mayores niveles de masificación y diversificación de estudiantes en países con mayores niveles de desarrollo, entre otros, a un acceso más equitativo de estudiantes de diferentes quintiles de ingreso. En el caso del estudiante hondureño, los resultados muestran que la mayoría de los estudiantes provienen de los quintiles I al III, es decir, los de menores ingresos, y una minoría representa a los quintiles IV y V. Esto podría ser consecuencia de una creciente desigualdad en el país y de tasas bajas de cobertura y graduación del nivel de educación medio.

Circunstancias de vida y estudiantes de maestría y distancia:

- Solo el $1.2 \%$ de los estudiantes manifestó tener una discapacidad, un porcentaje muy inferior al $8 \%$ que representa el total del país. 
- Los estudiantes de maestría y a distancia, como habría de esperarse, presentan perfiles un poco diferentes a los estudiantes de pregrado. Para iniciar, el estudiante de posgrado tiene un promedio de edad de 32 años. Además, se caracteriza por vivir más con su cónyuge y menos con sus padres, tiene mejores ingresos económicos familiares y existe un mayor número de estudiantes que trabajan en comparación con el estudiante de pregrado. El estudiante a distancia también se caracteriza por trabajar a tiempo completo, tiene más hijos y sus ingresos familiares son menores que los de otras jornadas.

- De acuerdo con lo mencionado anteriormente, podríamos deducir entonces, que el estudiante de pregrado de la zona urbana, es decir, de las principales sedes de las IES son los que tienen familias con mayor poder adquisitivo, un menor número de hijos y tienen más tiempo para sus estudios porque no trabajan la misma cantidad de horas que el estudiante de distancia.

- Lo anterior es un reflejo de las condiciones económicas y sociales del país, en el cual existe mayor pobreza en las zonas rurales, menores ingresos económicos y una mayor tasa de natalidad.
- El futuro de los estudiantes, según su propia percepción, es visto de mejor manera en el mundo laboral extranjero. Los deseos de seguir estudiando en el país o en el extranjero son casi iguales para los estudiantes, pero el anhelo de obtener un empleo supera al de seguir estudiando.

Finalmente, la literatura también nos dice que hay diferentes preferencias de aprendizaje de acuerdo con el perfil del estudiante de la actualidad. Por ejemplo, el estudiante presencial de tiempo completo podría preferir un ambiente tradicional de aula de clase, en el cual realiza interacción con el docente y sus compañeros. Sin embargo, para el estudiante que trabaja y/o el estudiante a distancia el aprendizaje se llevaría a cabo de manera mixta (videos y otros medios digitales) con un aula de clase o en algunos casos, ya de manera $100 \%$ digital. Adicionalmente, se establece que la modalidad mixta es la preferida por el estudiante de la generación $\mathrm{Z}$, es decir, el que está haciendo su ingreso actualmente a la universidad. Es necesario, entonces, dar un seguimiento a estos estilos de aprendizaje en el caso de Honduras de acuerdo con el estudiante de la cohorte actual y las de un futuro cercano.

Los cambios en el perfil del estudiante hondureño obligan a reflexionar en cómo adecuar la educación superior de acuerdo con 
estos nuevos retos. Entre los elementos que se pueden considerar están los siguientes:

- Establecer las diferencias entre los estudiantes que estudian a tiempo completo y los que trabajan a fin de brindar a estas últimas modalidades alternativas a la presencial, como es el caso de los cursos en línea.

- Desarrollar metodologías de enseñanza que combinen clases presenciales con el uso de la tecnología a fin de optimizar la labor fuera del aula, especialmente para los estudiantes a distancia $y / o$ que trabajan y cuyos estilos de aprendizaje podrían asimilar o adaptarse mejor a estos formatos.

- Priorizar la ayuda financiera o flexibilizar métodos de pagos a los estudiantes que trabajan $\mathrm{y} / \mathrm{o}$ a los estudiantes de la modalidad a distancia.

- Elaborar otras investigaciones que profundicen en estos y otros aspectos de las características de los estudiantes de educación superior.

\section{REFERENCIAS}

Centro Interuniversitario de Desarrollo (2016). Educación Superior en Honduras.

Santiago, Chile
Centro Interuniversitario de Desarrollo (2016). Educación Superior en Iberoamérica. Santiago, Chile

Dirección de Educación Superior (2018). La Educación Superior en Honduras y sus Líneas Estratégicas de Desarrollo. Tegucigalpa.

Ferreyra, María Marta, Ciro Avitabile, Javier Botero Álvarez, Francisco Haimovich Paz, and Sergio Urzúa. (2017). At a Crossroads: Higher Education in

Latin America and the Caribbean. Directions in Development. Washington, DC: World Bank.

Gwosć, Hauschildt, Vogtle (2016). Eurostudent VI (2016-2018): Social and Economic Conditions of Student Life in Europe. Alemania

Instituto Nacional de Estadística (2017). Encuesta Permanente de Hogares de Propósitos Múltiples. Tegucigalpa, Honduras Lopez, V. \& Moncada, G (s.f.). Equidad en el acceso y permanencia a educación superior en honduras para estudiantes originarios de pueblos indígenas y afrodescendiente. Caso UNAH, 2012 - 2015. Tegucigalpa, Honduras

Rama (2007). Los Nuevos Estudiantes en Circuitos Diferenciados de Educación. UDUAL. México 
Zuniga, Marco A. (1987). La Educación

Superior en Honduras. CRESALC-UNESCO.

Caracas

\section{Referencias electrónicas}

National Center for Education Statistics

(2018). College and University Education.

Fast Facts. Recuperado de:

https://nces.ed.gov/fastfacts/display.asp?id=372

Novella, R, Rucci, G (2018). Millenials en América Latina y el Caribe: ¿Trabajar o Estudiar? Banco Interamericano de Desarrollo. Recuperado de www.iadb.org/millennials

Selingo, Jeff (2018). The Future Learners. An Innovative Approach to Understanding the Higher Education Market and Building a Student-Centered University. Recuperado de: https://www.pearson.com/corporate/aboutpearson/innovation/future-learners.html

UNAH (2014). Informe Resultados PAA año 2014 DSA-UNAH. Recuperado de http://www.admisiones.unah.edu.hn/Publica ciones.aspx

UNESCO (2017). Global Education Monitoring Report. Accountability in Education: Meeting our Commitments. Recuperado de: http://unesdoc.unesco.org/images/0025/002 593/259338e.pdf

U.S. Department of Education, National Center for Education Statistics. (2018).
National postsecondary student aid study, for 2007-08 and 2015-16 cohorts. Recuperado de: https://nces.ed. gov/s u r v e y s/npsas/ 